\title{
A Giant retroperitoneal liposarcoma encasing entirely the right kidney: A case report and a review of nephrectomy indications
}

\author{
Houssem Harbi ${ }^{1}$, Amira Akrout ${ }^{2}$, Mohamed Fourati ${ }^{3}$, Amine Zouari ${ }^{2}$, and Nozha Toumi ${ }^{3}$ \\ ${ }^{1}$ Habib Bourguiba Hosp \\ ${ }^{2}$ Habib Bourguiba Hospital \\ ${ }^{3}$ University of Sfax Faculty of Medecine of Sfax
}

November 13, 2021

\begin{abstract}
A 68-year-old female was operated for a giant dedifferentiated retroperitoneal liposarcoma (RLS) encasing entirely the right kidney. She had an en-bloc resection of the tumor with right nephrectomy. The en-bloc resection should avoid R1 resection margins. Renal conservation is suggested if the kidney is widely displaced and for elderly patient.
\end{abstract}

\section{Introduction:}

Fifteen percent of sarcomas are located in the retroperitoneal space[1] . Liposarcoma is a rare malignant tumor that originates from primitive mesenchymal cells rather than from mature adipose tissue. It is the most common subtype of retroperitoneal sarcomas[2] .

Retroperitoneal liposarcoma (RLS) may reach a very large size and may cause tumoral infiltration of adjacent organs and large vessels before being diagnosed. The average diameter of the tumor is $20-25 \mathrm{~cm}$ with an average weight of 15 to $20 \mathrm{Kg}$. The complete resection of the mass and a favorable histopathologic grade are positively associated with long-term disease-free survival [2]. However, preserving neighboring structures, in particular the kidney, is actually non-consensual and still challenging. Thus, nephrectomy should be discussed case by case.

We report herein a case of a 68-year-old female with a giant retroperitoneal liposarcoma arising from the right perirenal space.

\section{Case report:}

A 68-year-old female with no medical history presented with a complaint of an abdominal painless mass. She has no associated urinary or digestive signs (as nausea, vomiting, dyspepsia or constipation). Physical examination showed a large hard non-mobile non-tender mass in the right hemi abdomen and extending from the subhepatic region down to the right pelvis. Laboratory findings and tumor markers were within the normal range. Computed tomography showed a voluminous retroperitoneal mass of $26 \times 14 \mathrm{~cm}$ encasing entirely the right kidney (figure 1). It had a mixed content, mainly of fat density and some microcalcifications. The intestinal loops and other abdominal organs were displaced to the midline without any signs of tumoral invasion. The Ct-scan didn't show any liver or pulmonary metastasis.

The patient underwent a midline laparotomy: a bulky, well-encapsulated mass was found arising from the right perinephric area and englobing entirely the right kidney. The tumor measuring $20 \mathrm{x} 30 \mathrm{x} 15 \mathrm{~cm}$ was totally excised en-bloc with the right kidney (figure 2). The radical nephrectomy was necessary to achieve clear macroscopic resection margins without any tumoral capsule break-in. 
The patient had an uncomplicated postoperative course and was discharged to home on the $6^{\text {th }}$ postoperative day.

Histological and immunohistochemistry analysis of the tumor revealed a dedifferentiated RLS with no evidence of tumor invasion of the kidney. Then, the patient had an adjuvant radiotherapy. After 18 months follow-up, the patient has no clinical or radiological signs of disease recurrence.

\section{Discussion:}

The RLS is a rare mesenchymal tumor representing $0.07 \%$ to $0.2 \%$ of all neoplasia. This tumor mainly affects people aged 40 to 60 years and its prevalence seems equivalent in both sexes [3] .

This tumor generally remains asymptomatic and can reach a large size (over $30 \mathrm{~cm}$ ) with possible invasion or displacement of neighboring organs. Indeed, the diagnosis is suggested when there is an abdominal mass syndrome, a significant increase in abdominal volume or when there are signs of digestive or urinary compression. This is explained by the fact that the retroperitoneum is a large expandable space without any bony boundaries $[4,5]$.

Differential diagnosis of RLS includes tumors with fatty components such as renal angioleiomyolipoma, adrenal myelolipoma, retroperitoneal lipoma, and teratoma [1] .

There are four histologic subtypes of liposarcoma: Undifferentiated, pleomorphic, well-differentiated and myxoid. The undifferentiated and pleomorphic types are associated to a high degree of malignancy[6] .

Computed tomography $(\mathrm{CT})$ is the most common used imaging method for the diagnosis and preoperative evaluation of RLS. Indications for Magnetic resonance imaging are Musculo-skelettal involvement or pelvic extension[6] .

Surgery is currently the only potentially curative treatment. In fact, complete surgical resection is the cornerstone of non-metastatic RLS treatment. Furthermore, R0 resection is the most consistent prognostic factor [5] .

It is usually technically challenging to achieve clear resection margins. In most cases, it is necessary to proceed to an en-bloc resection including one or more organs with the RLS [7]. This means that surgeons tend to resect certain organs, especially the kidney, in the case of giant RLS, to avoid difficult dissection and mainly R1 resection margins [5]. For these reasons, our patient had an en-bloc resection of the RLS with the right kidney.

The only definite indication for nephrectomy is kidney invasion or perirenal fat infiltration. This should at best be suspected on preoperative imaging and must be confirmed intraoperatively[8-10] .

A RLS tumor that encases the kidney is a relative indication for nephrectomy. In this case, kidney conservation may be attempted but there is always a risk of incomplete resection $[\mathbf{1 1}, \mathbf{1 2}]$. Some authors reported an en-bloc resection of the RLS with kidney and showed that there was no histologic tumoral involvement of the kidney $[\mathbf{1 3}, \mathbf{1 4}]$ as was the case of our patient. This means that nephrectomy could be avoided.

The absence of tumor (RLS) capsule break-in is a crucial condition for kidney conservation [15] . Avoiding nephrectomy seems also possible when the RLS is widely displacing the kidney $[4, \mathbf{1 6}]$. In addition to these criteria, the patient's advanced age is another argument for trying to preserve the kidney [11] .

An ureter encasement is not a formal indication for nephrectomy. In fact, a thorough dissection can be attempted with a prior insertion of a double $\mathrm{J}$ catheter to reduce the risk of a possible ureteral injury. Ureteral tumoral infiltration must lead to its resection with renal auto-transplantation or to an ureterostomy $[4,17,18]$.

The long-term prognosis of RLS with incomplete resection (R1) is poor with 5- and 10-years average survival rates at $16.7 \%$ and $8 \%$ respectively [5] .

Metastatic and/or unresectable RLS (invading vital structures) have obviously a very poor prognosis. 
Neoadjuvant therapy could be an option to downstage the tumor before surgical intervention. Patients with positive surgical margins or high grade liposarcoma also need the consolidation from adjuvant therapy. Studies have shown that radiotherapy reduces the risk of local recurrence and improves the recurrence-free interval. However, data supporting positive impact of radiotherapy on overall survival is limited [19] . At present the role of chemotherapy in the management of RLS remains controversial [3] .

Multidisciplinary cooperation is particularly important in the treatment of giant retroperitoneal liposarcoma. Long-term follow-up CT scan is important, especially within 2 years after initial resection, which is peak recurrence period [5] .

\section{Conclusion:}

The RLS remains a rare tumor and should be suggested in cas of any large retroperitoneal tumor. It is an aggressive tumor with no typical presentation. The complete resection of the mass and a favorable histopathologic grade are positively associated with long-term disease-free survival. The complete (R0) surgical resection needs frequently an en-bloc resection of the tumor with ipsilateral involved or encased organs in particular the kidney. Criteria in favor of renal conservation are well encapsulated RLS, widely displaced kidney, well differentiated RLS sub-type and patient advanced age. An entirely encased kidney and ureteral invasion are not formal indications for associated nephrectomy.

\section{References:}

1. Nikolaos S Salemis 1, Evangelos Tsiambas, Andreas Karameris, Efstathios Tsohataridis. Giant Retroperitoneal Liposarcoma with Mixed Histological Pattern: A Rare Presentation and Literature Review. J Gastrointest Cancer. 2009;40(3-4):138-41.

2. Hyun Ho Han, Kyung Hwa Choi, Dong Suk Kim, Woo Jin Jeong, Seung Choul Yang, Seon Jung Jang1, Jun Jeong Choi1, Woong Kyu Han. Retroperitoneal Giant Liposarcoma. Korean J Urol. 2010 Aug;51(8):579-82.

3. Chi Xu, Zhiqiang Ma, Hui Zhang, Jianchun Yu, Shuguang Chen. Giant retroperitoneal liposarcoma with a maximum diameter of $37 \mathrm{~cm}$ : a case report and review of literature. Ann Transl Med. 2020 Oct;8(19):1248

4. Olivia J McCallum 1, James J Burke 2nd, Alex J Childs, Alex Ferro, Donald G Gallup Retroperitoneal liposarcoma weighing over one hundred pounds with review of the literature. Gynecol Oncol. 2006 Dec;103(3):1152-4.

5. Xiangyu Zenga, Weizhen Liua, Xiuli Wub, Jinbo Gaoa, Peng Zhanga, Xiaoming Shuaia, and Kaixiong Taoa. Clinicopathological characteristics and experience in the treatment of giant retroperitoneal liposarcoma: A case report and review of the literature. Cancer Biol Ther 2017 Sep 2;18(9):660-665.

6. Montenegro A, Varas M, SánchezVizcaíno E, Naval J, Loras C, Abad R. A giant retroperitoneal liposarcoma with renal involvement: A case report and literature review. Gastroenterol Hepatol. 2019 Oct;42(8):490-491.

7. V Tonini, A Ussia, M Cervellera. En bloc resection of giant retroperitoneal liposarcoma involving the right colon - a video vignette. Colorectal Dis. 2020 Nov;22(11):1772-1773. doi: 10.1111/codi.15163.

8. Wei-Dong Zhang, DA-Ren Liu, Ri-Sheng Que, Chuan-Biao Zhou, Chen-Ni Zhan, Jian-Gang Zhao, L I Chen. Management of retroperitoneal liposarcoma: A case report and review of the literature. Oncol Lett. 2015 Jul;10(1):405-409. doi: 10.3892/ol.2015.3193.

9. Caizzone A, Saladino E, Fleres F, Paviglianiti C, Iaropoli F, Mazzeo C, Cucinotta E, Macrì A. Giant retroperitoneal liposarcoma: Case report and review of the literature. Int J Surg Case Rep. 2015;9:23-6. doi: 10.1016/j.ijscr.2015.02.019.

10. Lam-Chung CE, Rodríguez-Orihuela DL, Arízaga-Ramírez R, Almeda-Valdés P, Castillo-Valdez AK, Magaña-Pérez K, Ventura-Gallegos JL, Gamboa-Domínguez A, De Anda González J, Gómez-Pérez FJ, Cuevas-Ramos D. Acromegaly and a giant retroperitoneal liposarcoma producing igf-1. AACE Clin Case Rep. 2020 Apr 3;6(4):e165-e169. doi: 10.4158/ACCR-2020-0061.

11. Oh SD, Oh SJ, Suh BJ, Shin JY, Oh CK, Park JK, Kim YM, Kim BM. A Giant Retroperitoneal Liposarcoma Encasing the Entire Left Kidney and Adherent to Adjacent Structures: A Case Report. 
Case Rep Oncol. 2016 Jun 27;9(2):368-72. doi: 10.1159/000447488.

12. Wen SC, Lin C. Kidney displaced by giant retroperitoneal li-posarcoma in HIV patient. Int Braz J Urol. 2020 Jul-Aug;46(4):673-675. doi: 10.1590/S1677-5538.IBJU.2019.0515.

13. Almas T, Ullah M, Ehtesham M, Akbar A, Khan MK. En Bloc Resection of a Giant Retroperitoneal Liposarcoma: A Surgical Challenge. Cureus. 2020 Jun 20;12(6):e8730. doi: 10.7759/cureus.8730.

14. Hashimoto Y, Hatakeyama S, Tachiwada T, Yoneyama T, Koie T, Kamimura N, Yanagisawa T, Hakamada K, Ohyama C. Surgical treatment of a giant liposarcoma in a Japanese man. Adv Urol. 2010;2010:943073. doi: 10.1155/2010/943073.

15. De Nardi P, Bissolati M, Cristallo M, Staudacher C. Recurrent giant liposarcoma of the spermatic cord. Urology. 2012 Jan;79(1):113-4. doi: 10.1016/j.urology.2011.02.004.

16. El-Helou E, Alimoradi M, Sabra H, Naccour J, Haddad MM, Bitar H. Recurrent giant retroperitoneal liposarcoma with 10 years follow up. Case report and review of literature. Int J Surg Case Rep. 2020;75:504-512. doi: 10.1016/j.ijscr.2020.09.143.

17. S Yol 1, S Tavli, L Tavli, M Belviranli, A Yosunkaya. Retroperitoneal and scrotal giant liposarcoma: report of a case. Surg Today 1998;28(3):339-42. doi: 10.1007/s005950050136.

18. Bansal VK, Misra MC, Sharma A, Chabbra A, Murmu LR. Giant retroperitoneal liposarcoma- renal salvage by autotransplantation. Indian J Surg. 2013 Apr;75(2):159-61. doi: 10.1007/s12262-012-0474-z.

19. Hazen B, Cocieru A. Giant Retroperitoneal Sarcoma. J Gastrointest Surg. 2017 Mar;21(3):602-603. doi: 10.1007/s11605-016-3258-0.

\section{Figure legends:}

Figure 1: Computed tomography (with medium contrast injection) showing a giant retroperitoneal liposarcoma (blue star) encasing entirely the right kidney that is also displaced widely to the midline

Figure 2: Removed surgical specimen: the retroperitoneal liposarcoma was resected en-bloc with the right kidney (yellow star).
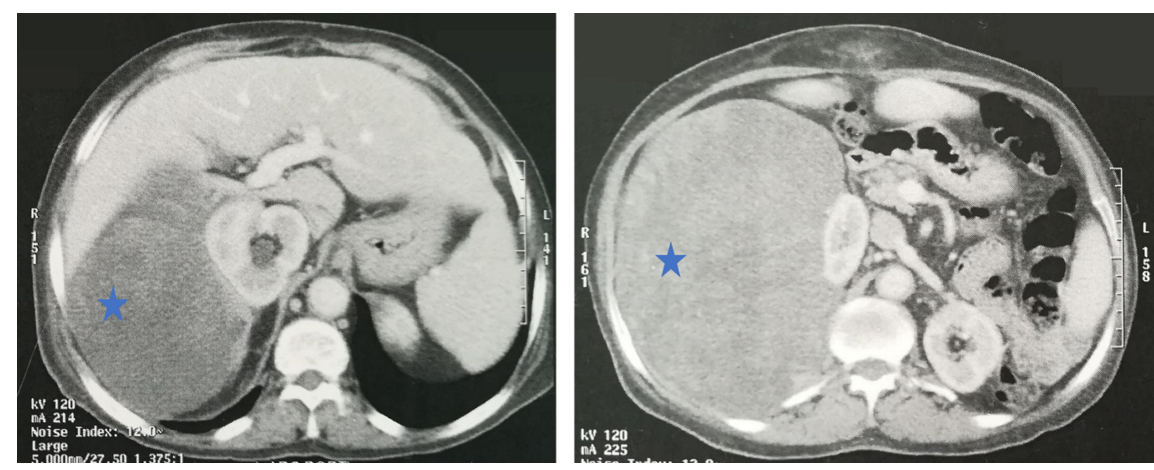

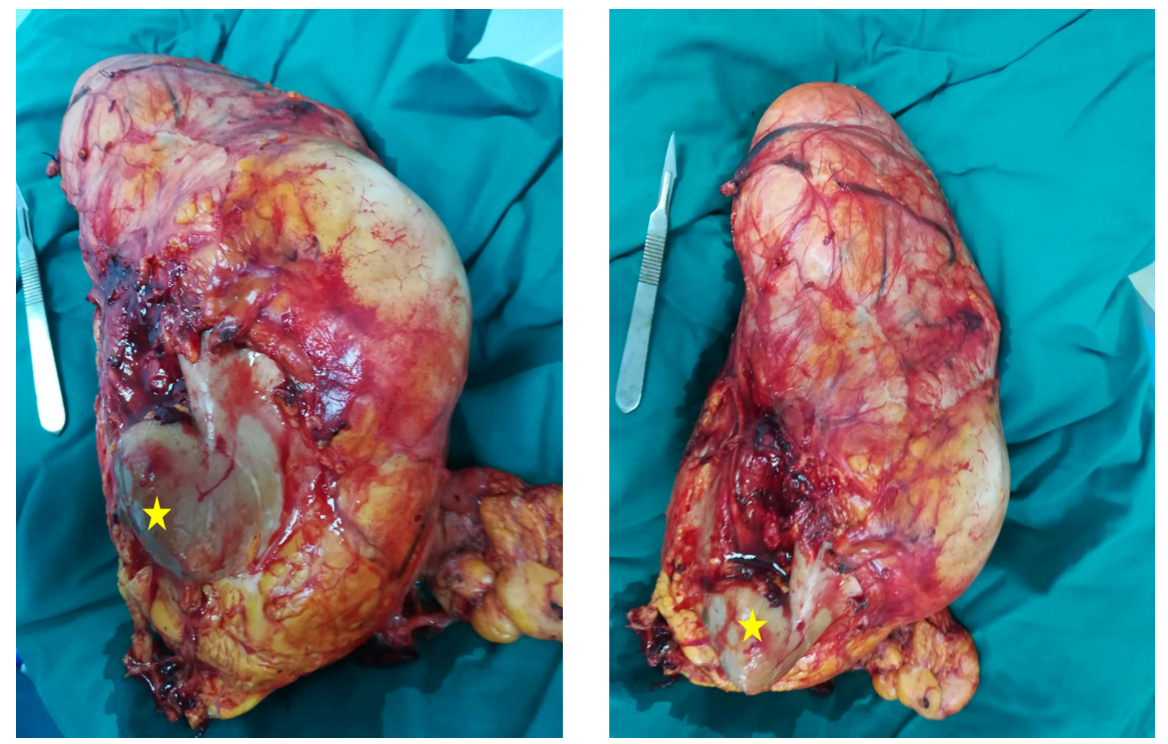